\title{
Heterogeneous Network Localization with a Distributed Phased Array Composed of Cooperative Vehicles
}

\author{
Siwei Zhang, Robert Pöhlmann and Armin Dammann \\ Institute of Communications and Navigation, German Aerospace Center (DLR) \\ 82234 Wessling, Germany \\ (e-mail: firstname.lastname@dlr.de)
}

\begin{abstract}
The emerging 5G technology provides direct data transmission, precise synchronization and localization for future autonomous vehicles. These vehicles are coupled in certain groups and drive simultaneously to enhance the road capacity and energy efficiency. The estimation of formation, i.e. the position of each individual vehicle w.r.t. the group, from the 5G signal is precise enough, so that the antennas deployed on multiple vehicles can be collectively considered as a distributed virtual antenna array. Non-cooperative entities, e.g. 5G incompatible vehicles, pedestrians or other road users, with radio transmissions according to a previous standard, can be precisely localized relative to the formation by distributed near-field array processing. By jointly locating the targets, the formation estimate of the $5 \mathrm{G}$ enabled vehicles is further improved.
\end{abstract}

\section{INTRODUCTION}

Ubiquitous realtime location information plays an essential role for future autonomous vehicular networks. These vehicles are coupled in certain formations and drive simultaneously to enhance the road capacity and energy efficiency [1]. A vehicle in these networks navigates autonomously according to the relative positions of itself and the neighboring objects like pedestrians and other vehicles. Traditionally, the relative location of a vehicle is estimated by fusing ego sensors such as inertial measurement units (IMUs) and odometer, relative sensors for example radar and optical sensors and absolute sensors like global navigation satellite system (GNSS) receivers. Terrestrial wireless communication networks offer additional opportunities to increase the localization reliability [2], especially with direct device-to-device (D2D) communications. A variety of topics related to terrestrial network localization have been covered in research. A framework of network localization is introduced in [3] and [4]. The coordinate system for network localization is discussed in [5] and [6]. Joint network localization and synchronization is investigated in [7]. In [8] and [9], the uncertain formation is exploited to locate external transmitters. Multiple vehicles are considered as a distributed phase array in [10] and [11] for angle of arrival (AoA) estimation.

The emerging $5^{\text {th }}$ generation mobile networks $(5 \mathrm{G})$ technology, especially millimeter wave (mmWave), provides reliable direct data transmission among the $5 \mathrm{G}$ enabled vehicles with a high carrier frequency and a large bandwidth [12]. Meanwhile, the $5 \mathrm{G}$ enabled vehicles are able to receive radio signals

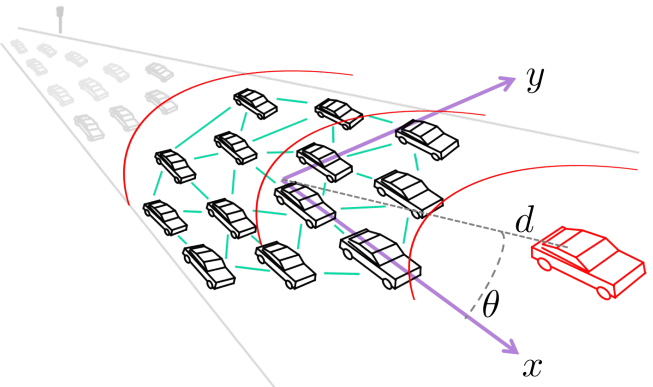

Figure 1: Heterogeneous network in vehicular application, where cooperative vehicles (agents) are illustrated in black and the non-cooperative vehicle (target) is indicated in red.

transmitted according to a previous wireless communication standard, e.g. intelligent transport systems (ITS)-G5, from non-cooperative entities like other vehicles. These signals are usually transmitted with a much lower carrier frequency and a smaller bandwidth compared to the $5 \mathrm{G}$ signals. We refer to a $5 \mathrm{G}$ enabled vehicle in the formation as an agent and a non-cooperative vehicle as a target. Targets do not exploit any signal for localization. The transceivers on all vehicles are considered neither carrier nor symbol synchronized.

In this work, we employ the Fisher information theory [13], [4] to investigate the potential of exploiting the heterogeneous radio access technologys (RATs) for simultaneous carrier/symbol synchronization, agent formation estimation and non-cooperative targets localization. Agents' position and clock information can be extracted from the symbol delays of the agent-to-agent (A2A) links. Due to a large A2A bandwidth, this information is precise enough, so that the antennas mounted on multiple agents can act collaboratively as a distributed phased array. Symbol delays and carrier phases of the target-to-agent (T2A) signals are jointly observed by this array and exploited for localization. The targets are located in the near-field of the distributed array's aperture, i.e. the T2A signal wavefronts are modeled as spherical waves. Not only the traditional AoA but also the distance of the targets w.r.t. the agent formation can be estimated [14] together with their clock and phase offsets. The accuracy of the target's location estimate is comparable with the agent formation estimate, since it is decisively determined by the 
carrier frequency of the T2A links. Therefore, agents can exploit the targets' location information to further improve their own position estimation, especially when multiple targets have been localized. A similar concept is introduced in [15], where external features are exchanged to improve network localization. Interestingly, even though a target does not actively cooperate with other vehicles, it indirectly benefits from other targets through an improved agent formation estimation. As a final result, the extended heterogeneous network is precisely synchronized and localized.

\section{SyStem MODEL}

We consider a network in two-dimensional (2D), for example as illustrated in Fig. 1, composed of $|\mathcal{M}|$ agents and $|\mathcal{S}|$ targets. An agent $\mathbb{a}_{u}$ is located at point $P_{u}$ and included in agent set $\mathcal{M}$. Agents in the network communicate to each other with a $5 \mathrm{G}$ bi-directional RAT. Agent $\mathbb{a}_{v}$ transmits a signal $s_{v}(t)$ with carrier frequency $f_{c}$ and bandwidth $B_{c}$, which is received by agent $\mathbb{a}_{u}$ through the A2A link $e_{u v}$ as

$$
r_{u v}(t)=\underbrace{\alpha_{u v} s_{v}\left(t-\tilde{\tau}_{u v}\right)}_{\triangleq s_{u v}(t)}+\epsilon_{u v}(t), \quad 0<t<T_{c},
$$

where $\alpha_{u v}$ is the positioning-irrelevant complex amplitude, $\tau_{u v}=d_{u v} / c_{0}$ is the signal propagation time, with the propagation speed $c_{0}$ and the Euclidian distance $d_{u v}$ between points $P_{u}$ and $P_{v}$. The observable delay with the clock at $\mathbb{a}_{u}$ is defined as $\tilde{\tau}_{u v}=\tau_{u v}+\delta_{v}-\delta_{u}$, where $\delta_{u}$ and $\delta_{v}$ are the clock offsets of $\mathbb{a}_{u}$ and $\mathbb{a}_{v}$. The additive noise $\epsilon_{u v}(t)$ is circularly-symmetric complex normally distributed with a variance $\sigma_{c}^{2} / 2$ for real and imaginary components respectively. Precise formation information may also be extracted from the A2A carrier phase with multiple antennas mounted on single agent [16]. In this work we only focus on the information contained in A2A symbol delay. Therefore a simplified single antenna model is employed. The total A2A link set is denoted as $\mathcal{E}_{c}$. Targets are located at points $P_{s}$ co-planar to the agent formation, $\forall \mathbb{a}_{s} \in \mathcal{S}$, with the target set defined as $\mathcal{S}$. Target $\mathbb{a}_{s}$ transmits a signal $s_{s}(t)$, with a carrier frequency $f_{s}$ and a bandwidth $B_{s}$ according to a previous wireless communication standard. Assume the $5 \mathrm{G}$ receiver at agents is backward compatible. Hence agents can receive the signal from $\mathbb{a}_{s}$ through the T2A links. The received signal at $\mathbb{a}_{u}$ through the T2A link $\mathbb{e}_{u s}$ within an observation window $0<t<T_{s}$ is expressed as

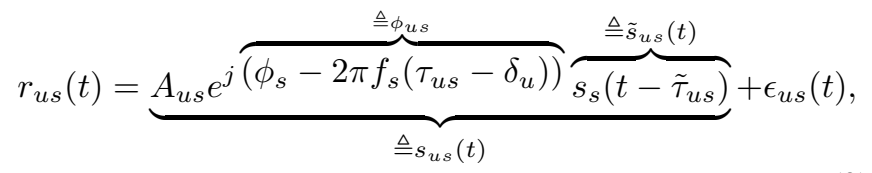

with the real-valued magnitude $A_{u s}$, carrier phases $\phi_{s}$ of $\mathbb{a}_{s}$. The delay observed with the clock at $\mathbb{a}_{u}$ is $\tilde{\tau}_{u s}=\tau_{u s}+\delta_{s}-\delta_{u}$. The noise $\epsilon_{u s}(t)$ is also circularly-symmetric complex normally distributed with a variance $\sigma_{s}^{2} / 2$ for real and imaginary components. We further assume the agents' phase offsets at the carrier frequency $f_{s}$ are adjusted to be aligned with the clock offset as $2 \pi f_{s} \delta_{u}$. The total T2A link set is denoted as $\mathcal{E}_{s}$. Agents perform anchor-free localization to jointly estimate the formation. The position estimates and their associated variances are subject to the choice of the coordinate system within the formation. An optimal 2D Cartesian coordinate system is defined by the arbitrary group motions [5], with the formation center as the origin. Similar to the group motions of the position estimates, clock offsets can be estimated only up to an arbitrary group offset. An optimal choice of the reference clock is to set the mean clock offset to zero. The impact of the reference system constraints on the Cramér-Rao bound (CRB) analysis will be discussed in Section IV. After the reference systems of position and clock are well defined, agent $\mathbb{a}_{u}$ 's coordinates are expressed as $\mathbf{p}_{u}=\operatorname{vec}\left\langle x_{u}, y_{u}\right\rangle$. The vector of unknown positioning relevant parameters of $\mathbb{a}_{u}$ is defined as $\mathbf{x}_{u}=\operatorname{vec}\left\langle\mathbf{p}_{u}, \delta_{u}\right\rangle$. The vectorizing operator $\operatorname{vec}\langle\cdots\rangle$ arranges elements into a column vector. The relevant unknowns in the formation are $\mathbf{x}_{\mathcal{M}}=\operatorname{vec}\left\langle\cdots, \mathbf{x}_{u}, \cdots\right\rangle, \forall a_{u} \in \mathcal{M}$. The position of a target w.r.t. the formation is represented with its polar coordinates $\mathbf{p}_{s}^{\mathcal{P}}=\operatorname{vec}\left\langle d_{s}, \theta_{s}\right\rangle$ in order to investigate the performances of distance and AoA estimations $\mathbb{a}_{s}$ individually. The corresponding Cartesian coordinates are $\mathbf{p}_{s}=d_{s} \operatorname{vec}\left\langle\cos \theta_{s}, \sin \theta_{s}\right\rangle$. The defined coordinate systems are illustrated in Fig. 1. The relevant unknowns of a target $\mathbb{a}_{s}$ and all targets are $\mathbf{x}_{s}=\operatorname{vec}\left\langle\mathbf{p}_{s}^{\mathcal{P}}, \delta_{s}, \phi_{s}\right\rangle$ and $\mathbf{x}_{\mathcal{S}}=$ $\operatorname{vec}\left\langle\cdots, \mathbf{x}_{s}, \cdots\right\rangle, \forall \mathbb{a}_{s} \in \mathcal{S}$. The total relevant unknowns in the extended heterogeneous network are $\mathbf{x}=\operatorname{vec}\left\langle\mathbf{x}_{\mathcal{M}}, \mathbf{x}_{\mathcal{S}}\right\rangle$. As indicated by (1) and (2), the location information of the agents and targets can be extracted from both A2A and T2A symbol delays, as well as the carrier phases of the T2A signals. Nodes are considered as isotropic points, which inherently applies the spherical wave model under the near-field assumption. We focus on the potential and scalability of vehicular network localization, and assume only line-of-sight (LOS) path propagation with the following justifications. Firstly, the considered D2D links are often in short range, where the LOS path is likely to be the dominant component. Secondly, mitigating multipath effects on localization is a well studied topic in both symbol delay [17] and carrier phase domain [18], which can be extended to our applications. Besides, we assume only a constant offset in clock uncertainty. In reality, the clock drift also affects the localization performance, which should be included as a nuisance parameter [7].

\section{FISHER INFORMATION IN NETWORK}

The parameters estimation covariance from an unbiased estimator is lower bounded by the CRB, which can be derived from the Fisher information matrix (FIM) [13]. The total FIM $\mathbf{J}$ is the superposition of the FIMs from the A2A links $\mathbf{J}_{\mathrm{a}}$ and the T2A links $\mathbf{J}_{\mathrm{s}}$ [4], i.e.

$$
\mathbf{J}=\mathbf{J}_{\mathrm{a}}+\mathbf{J}_{\mathrm{s}} .
$$


We define $\nabla_{\mathbf{a}^{T}} \mathbf{b} \in \mathbb{C}^{B \times A}$ as the derivatives of $\mathbf{b} \in \mathbb{C}^{B \times 1}$ w.r.t. $\mathbf{a} \in \mathbb{R}^{A \times 1}$. The FIM considering all $\mathrm{T} 2 \mathrm{~A}$ links is expressed as [19]

$$
\mathbf{J}_{\mathrm{s}}=\frac{4}{N_{0, s}} \Re\left\{\sum_{\mathbb{e}_{u s} \in \mathcal{E}_{s}} \int_{0}^{T_{s}} \nabla_{\mathbf{x}} s_{u s}^{*}(t) \nabla_{\mathbf{x}^{T}} s_{u s}(t) \mathrm{d} t\right\},
$$

where $N_{0, s}$ is the power spectral density (PSD) of the noise. The information of parameters $\mathbf{x}$ is in general contained in the signal phase $\phi_{u s}$ and delay $\tilde{\tau}_{u s}$, which can be stacked into a vector $\mathbf{u}_{u s}=\operatorname{vec}\left\langle\phi_{u s}, \tilde{\tau}_{u s}\right\rangle$. The integrand in (4) can be represented by the chain rule as

$$
\begin{aligned}
& \nabla_{\mathbf{x}} s_{u s}^{*}(t) \nabla_{\mathbf{x}^{T}} s_{u s}(t) \\
&= \nabla_{\mathbf{x}} \mathbf{u}_{u s}^{T} \nabla_{\mathbf{u}_{u s}} s_{u s}^{*}(t) \nabla_{\mathbf{u}_{u s}^{T}} s_{u s}(t) \\
&= \nabla_{\mathbf{x}^{T}} \mathbf{u}_{u s} \\
& \nabla_{\mathbf{x}} \mathbf{u}_{u s}^{T}\left[\begin{array}{cc}
\left\|\tilde{s}_{u s}(t)\right\|^{2} & -j \tilde{s}_{u s}^{*}(t) \frac{\partial \tilde{s}_{u s}(t)}{\partial \tilde{\tau}_{u s}} \\
j \tilde{s}_{u s}(t) \frac{\partial \tilde{s}_{u s}^{*}(t)}{\partial \tilde{\tau}_{u s}} & \left\|\frac{\partial \tilde{s}_{u s}(t)}{\partial \tilde{\tau}_{u s}}\right\|^{2}
\end{array}\right] \nabla_{\mathbf{x}^{T} \mathbf{u}_{u s} .}
\end{aligned}
$$

Consider the $N_{0, s} \rightarrow 0$ and $T_{s} \rightarrow \infty$ asymptotics [19]

$$
\int_{0}^{T_{s}}\left\|\tilde{s}_{u s}(t)\right\|^{2} \mathrm{~d} t=\int_{-\infty}^{\infty}\|S(f)\|^{2} \mathrm{~d} f \triangleq E_{u s} / A_{u s}^{2},
$$

with the received signal power $E_{u s}$,

$$
\begin{aligned}
& \int_{0}^{T_{s}} \tilde{s}_{u s}(t) \frac{\partial \tilde{s}_{u s}(t)^{*}}{\partial \tilde{\tau}_{u s}} \mathrm{~d} t \\
= & \int_{-\infty}^{\infty} \int_{-\infty}^{\infty} j 2 \pi f_{2} S\left(f_{2}\right) S^{*}\left(f_{1}\right) \\
\times & \int_{0}^{T_{s}} e^{j 2 \pi\left(f_{2}-f_{1}\right)\left(t-\tilde{\tau}_{u s}\right)} \mathrm{d} t \mathrm{~d} f_{1} \mathrm{~d} f_{2} \\
= & \int_{-\infty}^{\infty} \int_{-\infty}^{\infty} j 2 \pi f_{2} S\left(f_{2}\right) S^{*}\left(f_{1}\right) e^{j 2 \pi\left(f_{2}-f_{1}\right)\left(T_{s} / 2-\tilde{\tau}_{u s}\right)} \\
\times & \underbrace{\int_{T_{s} / 2}^{j 2 \pi\left(f_{2}-f_{1}\right) t^{\prime}} \mathrm{d} t^{\prime}}_{-T_{s} / 2} \mathrm{~d} f_{1} \mathrm{~d} f_{2} \\
= & j \int_{-\infty}^{\infty} 2 \pi f\|S(f)\|^{2} \mathrm{~d} f \triangleq j \gamma_{s} E_{u s} / A_{u s}^{2},
\end{aligned}
$$

with the centroid of the spectrum $\gamma_{s}$, and

$$
\begin{aligned}
& \int_{0}^{T_{s}}\left\|\frac{\partial \tilde{s}_{u s}(t)}{\partial \tilde{\tau}_{u s}}\right\|^{2} \mathrm{~d} t \\
= & \int_{-\infty}^{\infty} 4 \pi^{2} f^{2}\|S(f)\|^{2} \mathrm{~d} f \triangleq \beta_{s}^{2} E_{u s} / A_{u s}^{2},
\end{aligned}
$$

where $\beta_{s}$ is the root-mean-square bandwidth, or the effective bandwidth. The T2A-related FIM can be expressed as

$$
\mathbf{J}_{\mathrm{s}}=\sum_{\mathbb{e}_{u s} \in \mathcal{E}_{s}} \frac{4 E_{u s}}{N_{0, s}} \nabla_{\mathbf{x}} \mathbf{u}_{u s}^{T} \underbrace{\left[\begin{array}{cc}
1 & -\gamma_{s} \\
-\gamma_{s} & \beta_{s}^{2}
\end{array}\right]}_{\triangleq \mathbf{J}_{u s}} \nabla_{\mathbf{x}^{T}} \mathbf{u}_{u s} .
$$

The signal-to-noise ratio (SNR) is defined as $\mathrm{SNR}_{u s}=$ $2 E_{u s} / N_{0, s}$. Additionally if we assume a symmetric spectrum, i.e. $\gamma_{s}=0$, the matrix $\mathbf{J}_{\text {us }}$ becomes diagonal, which leads to maximal information of $\phi_{u s}$ and $\tilde{\tau}_{u s}$. The information contained in the A2A links can be derived in a similar fashion, which lead to the total FIM as

$$
\begin{aligned}
\mathbf{J} & =\mathbf{J}_{\mathrm{s}, \tau}+\mathbf{J}_{\mathrm{s}, \phi}+\mathbf{J}_{\mathrm{a}, \tau} \\
& =\sum_{\mathbb{e}_{u s} \in \mathcal{E}_{s}} \frac{4 E_{u s}}{N_{0, s}}\left(\beta_{s}^{2} \nabla_{\mathbf{x}} \tilde{\tau}_{u s} \nabla_{\mathbf{x}^{T}} \tilde{\tau}_{u s}+\nabla_{\mathbf{x}} \phi_{u s} \nabla_{\mathbf{x}^{T}} \phi_{u s}\right) \\
& +\sum_{\mathbb{e}_{u v} \in \mathcal{E}_{v}} \frac{4 E_{u v}}{N_{0, c}} \beta_{c}^{2} \nabla_{\mathbf{x}} \tilde{\tau}_{u v} \nabla_{\mathbf{x}^{T}} \tilde{\tau}_{u v},
\end{aligned}
$$

where $\mathbf{J}_{\mathrm{a}, \tau}, \mathbf{J}_{\mathrm{s}, \tau}$ and $\mathbf{J}_{\mathrm{s}, \phi}$ are the FIMs considering the symbol delays of the $\mathrm{A} 2 \mathrm{~A}$ and $\mathrm{T} 2 \mathrm{~A}$ links and the carrier phases of the T2A links, respectively. The partial derivatives required to calculate (10) are listed in the Appendix.

\section{CRAMÉr-RAo Bound With CONSTRAints}

For absolute localization and synchronization, the reference system is externally defined. In this case, the CRB is expressed by simply inverting the full-rank FIM, and is invariant to coordinate system transformations. On the contrary, for relative localization and synchronization problems, the FIM is rank-four deficient, since the localization solutions are subject to arbitrary group rotation and translation and the clock offset estimates are subject to arbitrary group offset. Reference systems have to be defined by the minimal sufficient constraints to the estimated parameters depending on the application. In [9] a baseline defined by two agents are used as the coordinate system, which is not optimal. In our heterogeneous network, we are interested in the accuracy of the formation estimation and the targets localization w.r.t. the formation. Therefore, an optimal Cartesian coordinate system is defined by constraining the group rotations and translations of the formation [6]. An optimal clock reference system of the formation is defined by setting the mean clock offset to a constant. The network/target localization and synchronization is employed w.r.t. the above mentioned reference system. The constrains of the reference system are represented by the subspace $\mathbf{U}_{\perp}=\left[\mathbf{u}_{x}, \mathbf{u}_{y}, \mathbf{u}_{\delta}, \mathbf{u}_{r}\right]$, with orthonormal bases of translations in $x$ and $y$ directions $\mathbf{u}_{x}$ and $\mathbf{u}_{y}$, clock offset $\mathbf{u}_{\delta}$ and rotation $\mathbf{u}_{r}$ defined as

$$
\begin{aligned}
& {\left[\mathbf{u}_{x}, \mathbf{u}_{y}, \mathbf{u}_{\delta}\right]=\frac{1}{\sqrt{|\mathcal{M}|}}\left[\mathbf{1}_{1 \times|\mathcal{M}|} \otimes \mathbf{I}_{3 \times 3}, \mathbf{0}_{3 \times 4|\mathcal{S}|}\right]^{T}} \\
& \mathbf{u}_{r}=\frac{1}{\left\|\mathbf{p}_{\mathcal{M}}\right\|}\left[y_{1},-x_{1}, 0, \cdots, y_{|\mathcal{M}|},-x_{|\mathcal{M}|}, 0, \mathbf{0}_{1 \times 4|\mathcal{S}|}\right]^{T}
\end{aligned}
$$

The bases $\mathbf{U}_{\perp}$ spans the left nullspace of the problem, i.e. the reference constraints. The orthonormal bases of column space 


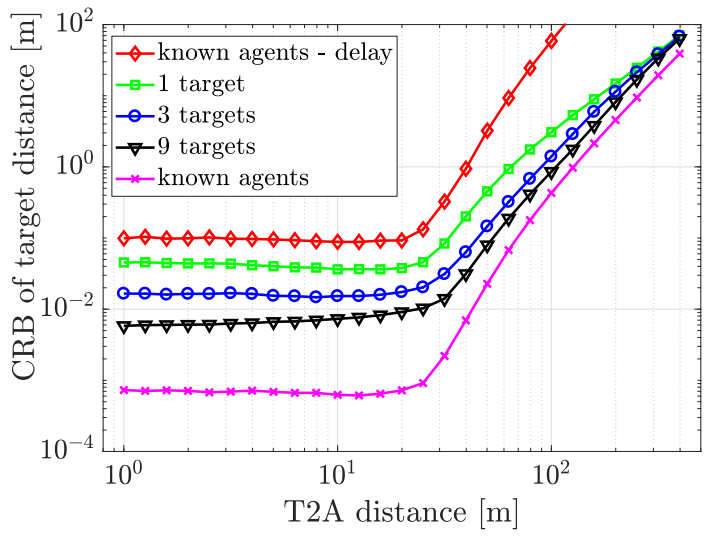

Figure 2: CRBs of target distance estimation.

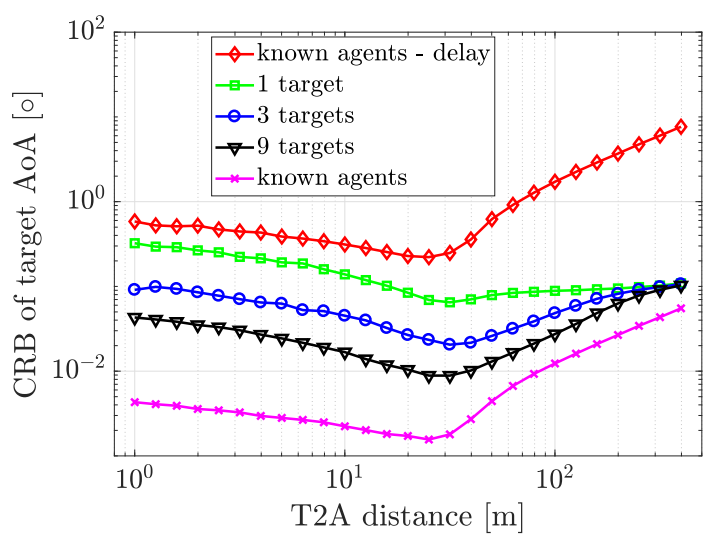

Figure 3: CRBs of target AoA estimation.

$\mathbf{U}_{\|}$can be determined by the Eigen-decomposition as

$$
\mathbf{I}-\mathbf{U}_{\perp} \mathbf{U}_{\perp}^{T}=\left[\mathbf{U}_{\|}, \tilde{\mathbf{U}}_{\perp}\right]\left[\begin{array}{cc}
\boldsymbol{\Lambda} & \mathbf{0} \\
\mathbf{0} & \mathbf{0}
\end{array}\right]\left[\begin{array}{c}
\mathbf{U}_{\|}^{T} \\
\tilde{\mathbf{U}}_{\perp}^{T}
\end{array}\right] .
$$

The total FIM can be projected onto the column space as $\mathbf{U}_{\|}^{T} \mathbf{J} \mathbf{U}_{\|}$and becomes full-rank. Finally, the CRB of $\mathbf{x}$ is calculated by inverting the projected FIM and transforming back to the parameter space as

$$
\mathrm{CRB}[\mathbf{x}]=\mathbf{U}_{\|}\left(\mathbf{U}_{\|}^{T} \mathbf{J} \mathbf{U}_{\|}\right)^{-1} \mathbf{U}_{\|}^{T},
$$

where the variance of a parameter estimate $\operatorname{var}\left[[\hat{\mathbf{x}}]_{i}\right]$ is lower bounded by

$$
\operatorname{var}\left[[\hat{\mathbf{x}}]_{i}\right] \geqslant[\mathrm{CRB}[\mathbf{x}]]_{i, i}
$$

\section{NumericAl Results}

We conduct simulations to demonstrate the potential of the heterogeneous network localization with the agent formation as a phased array. Assume 30 cooperative vehicles (agents) spread over a length of $90 \mathrm{~m}$ on a $30 \mathrm{~m}$-wide motorway. The vehicles' positions are uniformly generated 1000 times within the area, which averages out impacts from any particular formation. The signals propagating on A2A links use a carrier frequency of $f_{c}=60 \mathrm{GHz}$ and a bandwidth of $B_{c}=1 \mathrm{GHz}$.

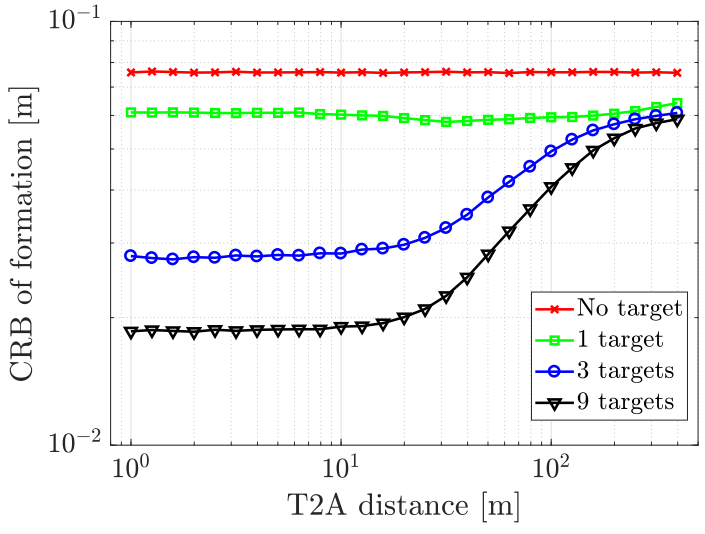

Figure 4: CRBs of formation estimation RMSE.

A free-space pathloss model is assumed with an SNR of $0 \mathrm{~dB}$ at distance $10 \mathrm{~m}$. Different groups of non-cooperative vehicles (targets) are deployed on the same motorway, with a number varying from zero to nine and a distance to the agent formation's center from $1 \mathrm{~m}$ to $400 \mathrm{~m}$. The non-cooperative vehicles are transmitting T2A signals with a carrier frequency of $f_{s}=1 \mathrm{GHz}$ and a bandwidth of $B_{s}=25 \mathrm{MHz}$, which are only received by the agents. A similar free-space pathloss is assumed with an SNR of $0 \mathrm{~dB}$ at distance $316 \mathrm{~m}$. All the agents and targets are neither carrier nor symbol synchronized. The symbol delays of the A2A and T2A links and the carrier phases of the T2A links are exploited for estimating the formation and locating the targets. We also consider two cases where agents' locations and clock offsets are perfectly known as benchmarks. The perfect agents' knowledge assumption is valid when the number of targets approaches infinity, or the formation is precisely estimated with other measurements, e.g. from radar, optical sensors or the phased array for A2A signal. In the first benchmark case, only symbol delays of the $\mathrm{A} 2 \mathrm{~A}$ and $\mathrm{T} 2 \mathrm{~A}$ links are exploited. It is equivalent to the traditional target localization with time difference of arrival (TDoA) measurements. In the second case, carrier phases of the T2A links are considered additionally. The square root of CRBs of the target's distance and AoA estimation w.r.t. the formation are plotted in Fig. 2 and Fig. 3, respectively. The root mean CRBs of the agent formation estimation, i.e. the position error bound averaged over all agents, are illustrated in Fig. 4. First, the curves of two benchmarks have the same tendency with a fixed gap of $21.4 \mathrm{~dB}$ for both distance and AoA performances, which is due to the resolution difference between bandwidth and carrier frequency of the T2A signals. Second, the CRBs of targets' distance estimation are stable inside the formation, and increasing while targets are moving away from the formation. Third, the CRBs of target AoA estimation are decreasing in the cases of uncertain formations when the target move from center toward the formation border. It indicates that the AoA estimation is more sensitive to the formation uncertainty around the formation center. The AoA estimation CRBs increase when targets are outside of the 
formation due to a decreasing SNR similarly as the traditional far-field AoA estimation. Forth, all three plots show benefits of jointly observing multiple targets on both target localization and formation estimation, where the joint estimation performances are approaching to the second benchmark. These benefits get smaller when targets move away from the network due to the reduced SNR and the unfavorable geometry. Last but not least, the proposed joint symbol delay and carrier phase processing in the considered heterogeneous network has a promising potential. Sub-meter target distance estimation accuracy is achieved with a T2A distance up to one hundred meters. A $0.1^{\circ}$ target AoA estimation accuracy and a few centimeters formation estimation accuracy are guaranteed for all the evaluated T2A distances.

\section{CONCLUSION}

We have investigated the CRBs of localization in a heterogeneous asynchronous vehicular network, containing $5 \mathrm{G}$ enabled agents and non-cooperative targets with radio transmissions according to a previous standard. The agent formation and the targets' locations are jointly estimated from the symbol delays of both A2A and T2A signals as well as the carrier phase of the T2A signals. Both of the formation estimation and the target localization accuracies are significantly improved when more targets are included, even though the targets are not actively cooperating with other vehicles.

\section{APPENDIX}

Exploiting the inter-node distance expression in [14], the partial derivatives in (10) can be written as

$$
\begin{aligned}
-\nabla_{\mathbf{x}_{u}} \tilde{\tau}_{u v} & =\nabla_{\mathbf{x}_{v}} \tilde{\tau}_{u v}=\operatorname{vec}\left\langle\frac{\cos \theta_{u v}}{c_{0}}, \frac{\sin \theta_{u v}}{c_{0}}, 1\right\rangle, \\
\nabla_{\mathbf{x}_{u}} \tilde{\tau}_{u s} & =-\operatorname{vec}\left\langle\frac{\cos \theta_{u s}}{c_{0}}, \frac{\sin \theta_{u s}}{c_{0}}, 1\right\rangle, \\
\nabla_{\mathbf{x}_{s}} \tilde{\tau}_{u s} & =\operatorname{vec}\left\langle\frac{\nabla_{\mathbf{p}_{s}^{\mathcal{P}}} d_{u s}}{c_{0}}, 1,0\right\rangle, \\
\nabla_{\mathbf{x}_{u}} \phi_{u s} & =\operatorname{vec}\left\langle\frac{2 \pi f_{c} \cos \theta_{u s}}{c_{0}}, \frac{2 \pi f_{c} \sin \theta_{u s}}{c_{0}}, 2 \pi f_{c}\right\rangle, \\
\nabla_{\mathbf{x}_{s}} \phi_{u s} & =\operatorname{vec}\left\langle\frac{-2 \pi f_{c}}{c_{0}} \nabla_{\mathbf{p}_{s}^{\mathcal{P}}} d_{u s}, 0,1\right\rangle,
\end{aligned}
$$

where $\theta_{u v}$ and $\theta_{u s}$ are the angles of incoming signals $r_{u v}(t)$ and $r_{u s}(t)$, w.r.t. the formation coordinate system, and

$\nabla_{\mathbf{p}_{s}^{\mathcal{P}}} d_{u s}=\operatorname{vec}\left\langle\frac{d_{s}-d_{u} \cos \left(\theta_{s}-\theta_{u}\right)}{d_{u s}}, \frac{d_{u} d_{s} \sin \left(\theta_{s}-\theta_{u}\right)}{d_{u s}}\right\rangle$.

\section{REFERENCES}

[1] F. Dressler, F. Klingler, M. Segata, and R. L. Cigno, "Cooperative driving and the tactile internet," Proc. IEEE, vol. 107, no. 2, pp. 436446, Feb 2019.
[2] J. del Peral-Rosado, R. Raulefs, J. López-Salcedo, and G. SecoGranados, "Survey of cellular mobile radio localization methods: From $1 \mathrm{G}$ to 5G," IEEE Commun. Surveys Tuts., vol. 20, no. 2, pp. 1124-1148, 2018.

[3] J. Aspnes, T. Eren, D. K. Goldenberg, A. S. Morse, W. Whiteley, Y. R. Yang, B. D. O. Anderson, and P. N. Belhumeur, "A theory of network localization," IEEE Trans. Mobile Comput., vol. 5, no. 12, pp. 1663 1678, Dec. 2006.

[4] Y. Shen, H. Wymeersch, and M. Win, "Fundamental limits of wideband localization, part II: Cooperative networks," IEEE Trans. Inf. Theory, vol. 56, no. 10, pp. 4981-5000, Oct. 2010.

[5] J. N. Ash and R. L. Moses, "On the relative and absolute positioning errors in self-localization systems," IEEE Trans. Signal Process., vol. 56, no. 11, pp. 5668-5679, Nov. 2008.

[6] D. C. Popescu, M. Hedley, and T. Sathyan, "Posterior cramér-rao bound for anchorless tracking," IEEE Signal Process. Lett., vol. 20, no. 12, pp. 1183-1186, Dec 2013.

[7] B. Etzlinger, F. Meyer, F. Hlawatsch, A. Springer, and H. Wymeersch, "Cooperative simultaneous localization and synchronization in mobile agent networks," IEEE Trans. Signal Process., vol. 65, no. 14, pp. 35873602, July 2017.

[8] C. Taylor, A. Rahimi, J. Bachrach, H. Shrobe, and A. Grue, "Simultaneous localization, calibration, and tracking in an ad hoc sensor network," in Proceedings of the 5th International Conference on Information Processing in Sensor Networks, ser. IPSN '06. New York, NY, USA: ACM, 2006, pp. 27-33. [Online]. Available: http://doi.acm.org/10.1145/1127777.1127785

[9] S. Zhang, R. Raulefs, and A. Dammann, "Location information driven formation control for swarm return-to-base application," in $201624^{\text {th }}$ European Signal Processing Conference (EUSIPCO), Aug. 2016, pp. 758-763.

[10] W. Suleiman, A. A. Vaheed, M. Pesavento, and A. M. Zoubir, "Decentralized direction-of-arrival estimation for arbitrary array geometries," in 2016 24th European Signal Processing Conference (EUSIPCO), Aug 2016, pp. 1921-1925.

[11] D. Shutin and S. Zhang, "Distributed sparsity-based bearing estimation with a swarm of cooperative agents," in 2016 IEEE Global Conference on Signal and Information Processing (GlobalSIP), Dec 2016, pp. 555559.

[12] J. Choi, V. Va, N. Gonzalez-Prelcic, R. Daniels, C. R. Bhat, and R. W. Heath, "Millimeter-wave vehicular communication to support massive automotive sensing," IEEE Commun. Mag., vol. 54, no. 12, pp. 160167, December 2016

[13] S. M. Kay, Fundamentals of Statistical Signal Processing: Estimation Theory. Upper Saddle River, NJ, USA: Prentice-Hall, Inc., 1993.

[14] S. Zhang, T. Jost, R. Pöhlmann, A. Dammann, D. Shutin, and P. A. Hoeher, "Spherical wave positioning based on curvature of arrival by an antenna array," IEEE Wireless Commun. Lett., vol. 8, no. 2, pp. 504507, Apr. 2019.

[15] G. Soatti, M. Nicoli, N. Garcia, B. Denis, R. Raulefs, and H. Wymeersch, "Implicit cooperative positioning in vehicular networks," IEEE Trans. Intell. Transp. Syst., vol. 19, no. 12, pp. 3964-3980, Dec. 2018.

[16] A. Shahmansoori et al., "Position and orientation estimation through millimeter-wave MIMO in 5G systems," IEEE Trans. Wireless Commun., vol. 17, no. 3, pp. 1822-1835, Mar. 2018.

[17] D. Dardari, A. Conti, U. Ferner, A. Giorgetti, and M. Z. Win, "Ranging with ultrawide bandwidth signals in multipath environments," Proc. IEEE, vol. 97, no. 2, pp. 404-426, Feb. 2009.

[18] T. E. Tuncer and B. Friedlander, Classical and Modern Direction-ofArrival Estimation. Boston: Academic Press, 2009.

[19] H. V. Poor, An Introduction to Signal Detection and Estimation (2 $2^{\text {nd }}$ Ed.). Berlin, Heidelberg: Springer-Verlag, 1994. 